\title{
Determinação de Inibidores de Degradação em Polietileno de Alta Densidade (PEAD) Através da Extração por Micro-Ondas e Análise via HPLC
}

\author{
Augusto O. Cardoso
}

A extração e subsequente separação e quantificação de substâncias que retardam o processo de degradação no Polietileno de Alta Densidade (PEAD), denominadas como aditivos, têm provado ser um desafio para os químicos. Existem alguns métodos que utilizam o refluxo com solventes específicos para esta extração, assim como energia sônica, forno de microondas, extração com fluido supercrítico, apenas para citar os mais utilizados. Recentemente foi lançado no mercado um digestor que utiliza micro-ondas, normalmente, usado em sínteses orgânicas, mas que também pode ser utilizado nas extrações de aditivos em polímeros. Por ser uma técnica nova, na literatura praticamente não existem publicações científicas a respeito do seu uso para a extração de aditivos em PEAD, embora para polipropileno e polietileno linear já existam métodos consagrados para esta finalidade. Foi obtida uma boa condição para a extração de irganox 1010, irganox 1076 e irgafos 168 fosfito e fostato em 30 minutos, com subsequente separação em corrida de oito minutos, utilizando HPLC com fase reversa, coluna C18 e fase móvel isocrática composta por acetonitrila e metanol. Salientamos que, apesar de terem sido determinadas condições ótimas de extração, não significa afirmar que sejam as únicas possíveis. Certamente existirão outras combinações de parâmetros que trarão boas taxas de recuperação destes aditivos em PEAD.

\section{Palavras-chave: Rede Organizacional; Capital Intelectual; Método Multicritério.}

The extraction and subsequent separation and quantitation of the substances that inhibit de degradation process in Polyethylene of High Density (PEHD), classified as Additives, has proven to be a challenge for the chemist. Some methods have been used such as Reflux with specifics solvents, sonication, microwaves, super critic conditions among others. Recently was introduced in the market a Digestor that uses microwaves, normally used in organic synthesis, but with chances to be used for the extractions as well. As a new technique in the literature it is almost impossible to find articles about this subject for extraction of additivies in PEHD, although for the extraction in the linear PE and PP can be found some publications. It was determined a good way to extract Irganox 1010, Irgafos 168 and Irganos 1076 in only 30 minutes with subsequent analysis by HPLC with reverse phase, $\mathrm{C} 18$ column and the isocratic condition using Acetonitrile and Methanol. Must be emphasized that although it was found a good condition for the extraction and the analysis of additives in PEHD, for sure, that is not the only way of dealing with job in PEAD.

Keywords: Organizational Network; Intellectual Capital; Multicriteria Methods. 


\section{Introdução}

A análise de aditivos em Polietileno de Alta Densidade, PEAD, é uma atividade bastante complexa, devido à dificuldade da sua extração nesta matriz. O refluxo com apenas ciclo-hexano é muito usado, mas na técnica de micro-ondas, ele não é eficiente se for utilizado sozinho.

A extração de aditivos pode ser feita por refluxo de solvente aquecido (Soxhlet), ultrassom, fluido supercrítico, micro-ondas, etc. Neste estudo, os antioxidantes foram extraídos por dissolução em solvente, utilizando energia sônica, em aparelho comercialmente conhecido como Discover-SP.

Na maioria das poliolefinas, são adicionados aditivos que podem ser classificados como antioxidantes, estabilizadores UV, plastificantes, etc. Estes têm a finalidade de estabilizar o polímero, retardando as reações de degradação pela ação dos raios ultravioleta, calor, reações com o oxigênio ou até mesmo para modificar algumas de suas propriedades. Essas substâncias são adicionadas logo após a extrusão, para que haja redução na formação de aldeídos, cetonas, assim como, o desenvolvimento de coloração no produto.

A recuperação desses aditivos, em níveis superiores à $90 \%$,é desejável para que se tenha um processo eficiente de quantificação da aditivação. Foi selecionada e moída uma amostra de PEAD para a realização dos ensaios. Na Tabela 1, estão mostrados os aditivos estudados neste trabalho.

Os polímeros não seriam capazes de atender às diversas demandas, se não houvesse a adição de aditivos em sua estrutura. Sem a sua incorporação, alguns polímeros degradariam, oxidariam, ficariam quebradiços, perderiam ou desenvolveriam cor, perderiam peso molecular com o tempo, devido à oxidação, calor, radiação, exposição a metais ou produtos químicos. Polímeros do tipo polietileno são bastante susceptíveis à oxidação pelo ar atmosférico.

Tabela 1. Alguns antioxidantes utilizados em poliolefinas.

\begin{tabular}{|l|l|}
\hline ADITIVO & NOME QUÍMICO \\
\hline Irgafos 168 & $\begin{array}{l}\text { Tris (2,4-di-t-butylphenyl } \\
\text { phosphite) }\end{array}$ \\
\hline Irganox 1010 & $\begin{array}{l}\text { Tetrakis methylene } \\
\text { (3,5-di-t-butyl-4- } \\
\text { hydroxyhydrocinnamate) } \\
\text { methane }\end{array}$ \\
\hline Irganox 1076 & $\begin{array}{l}\text { Octadecyl-3-(3,5-di-t-butyl-4- } \\
\text { hydroxyphenyl) propionate }\end{array}$ \\
\hline
\end{tabular}

Os aditivos melhoram a performance e a estabilidade de muitas resinas. A maneira pela qual eles trabalham depende da classe do aditivo. Existem no comércio inúmeros aditivos sendo os mais comuns os antioxidantes primários e secundários, os retardantes de chama, neutralizadores de radicais ácidos e agentes de nucleação.

Radicais livres são gerados quando estes polímeros são expostos ao calor, radiação ultravioleta, esforço mecânico ou contato com impurezas metálicas. Estes radicais livres são extremamente reativos e podem promover a produção de mais radicais desse tipo e, a partir disso, pode ser desenvolvida uma série de reações em cadeia.

\section{Aditivos em Polímeros}

Oitenta por cento de todos os plásticos utilizados no mundo são termoplásticos. $\mathrm{Na}$ indústria alimentícia usualmente são utilizados o poliestireno, o polietileno e o cloreto de polivinila. Aditivos são introduzidos nesses polímeros para melhorar as suas propriedades mecânicas, estabilidade térmica, entre outras. Entretanto, tem sido reportado na literatura que a utilização destes aditivos pode gerar efeitos adversos na saúde humana e no meio ambiente (Groscalude, 1999; Lacaze, 1993) ${ }^{1,2}$. Já os antioxidantes secundários evitam a formação de mais radicais livres, através da decomposição de hidroperóxidos, antes que eles sofram uma cisão homolítica. Nesse caso, há a decomposição desses hidroperóxidos, formando produtos estáveis ${ }^{3-10}$.

São constituídos por moléculas contendo fósforo trivalente, que sofrem reações de hidrólise para estabilizar o polímero, tais como o irgafos 168. Importante haver um método rápido e preciso para analisar estes aditivos que são incorporados às poliolefinas.

Existem dois tipos de antioxidantes: os primários e os secundários. Os primários são definidos como os aditivos que interceptam e estabilizam os radicais livres através da doação de um átomo de hidrogênio ativo. Eles são doadores de prótons, constituídos por moléculas, contendo o grupo fenol na sua estrutura, por exemplo, o irganox 1010 e o irganox 1076. As suas estruturas e os respectivos espectros podem ser vistos nas Figuras 2 e 3. Eles trabalham muito bem sem a presença de outros aditivos. Radicais fenólicos ramificados e os de aminas 
aromáticas ramificadas, representam os dois principais tipos de antioxidantes primários.

A Tabela 1 apresenta alguns deles. Os mecanismos de ação dos radicais fenólicos ramificados e dos aminas são diferentes, sendo que os fenólicos são os mais utilizados.

Já os antioxidantes secundários evitam a formação de mais radicais livres através da decomposição de hidroperóxidos antes que eles sofram uma cisão homolítica. Neste caso, existe a decomposição destes hidroperóxidos, formando produtos estáveis.

São constituídos por moléculas contendo fósforo trivalente, que sofrem reações de hidrólise para estabilizar o polímero, tais como o irgafós 168 . Importante haver um método rápido e preciso para analisar estes aditivos que são incorporados às poliolefinas.

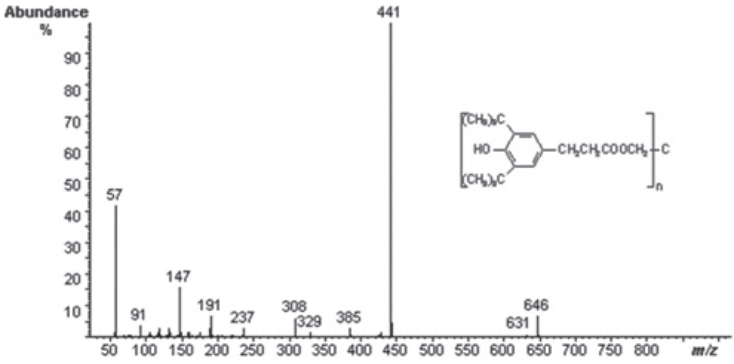

Figura 1. Fórmula química e espectro de massas do irganox 1010.

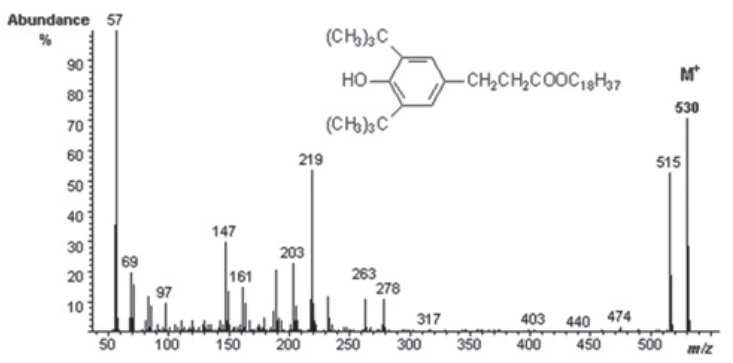

Figura 2. Fórmula química e espectro de massas do irganox 1076.

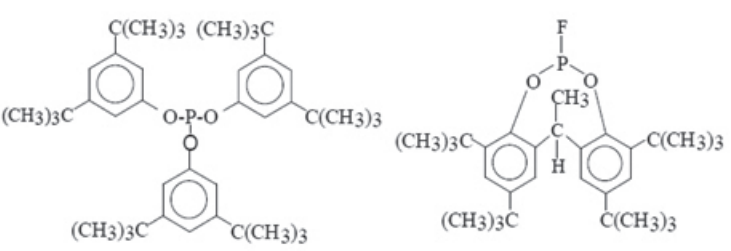

Irgafos 168

Ethanox 398

Figura 3. Estrutura química do irgafos 168 e do ethanox 398.

O mecanismo de ação é mostrado na Figura 4. Eles doam o seu hidrogênio fenólico ao radical gerado, estabilizando o polialcano. Nesse processo de estabilização, o radical alquil livre gera um grupamento fenoxi ramificado estável, possuindo uma estrutura do tipo quinona.

De um modo geral, o peso molecular da molécula do antioxidante está relacionada à temperatura de estabilidade dessa molécula. Por exemplo, o peso molecular do ethanox 330 é 775 e o do irganox 1076 é 531. O ethanox 330 tem maior estabilidade térmica do que o irganox 1076. Na Figura 3, podemos observar que a estrutura do ethanox 330 tem três radicais fenóis ramificados, enquanto que o irganox 1076 contém apenas um. Teoricamente, o ethanox 330 pode estabilizar três mais radicais do que o irganox 1076 .

Os antioxidantes secundários previnem a formação de radicais livres porque impedem que haja uma cisão homolítica na cadeia do hidroperóxido. Ao invés dessa cisão, ocorre uma estabilização dessa molécula. Consequentemente, eles ganham o nome de estabilizadores de peróxidos. A segunda função dos antioxidantes secundários é que eles também podem regenerar o antioxidante primário. Os dois principais tipos de antioxidantes secundários são os fosfitos e os tioésteres. Estes dois tipos trabalham da mesma maneira.

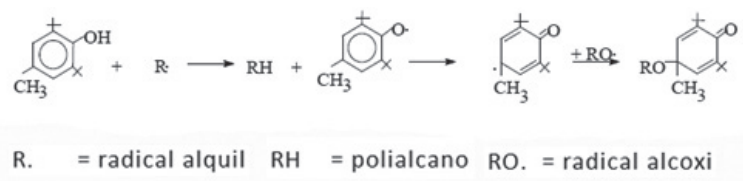

Figura 4. Mecanismo de ação dos radicais fenólicos. 
Tabela 2. Alguns antioxidantes secundários

\begin{tabular}{|c|c|c|}
\hline ADITIVO & TIPO & MOL \\
\hline Weston 399 & Aromático & 688 \\
\hline Irgafox 168 & Aromático & 647 \\
\hline Ethanox 398 & Fluoro & 487 \\
\hline
\end{tabular}

A Tabela 2 mostra alguns exemplos desses produtos. Eles operam melhor com a presença dos primários, porém, nem todo tipo de antioxidante primário pode ser misturado com o secundário. Os antioxidantes secundários trabalham melhor com a combinação correta em relação aos primários. Os fosfitos constituem o tipo mais comum de antioxidantes secundários. O mecanismo de atuação destes antioxidantes é mostrado na Figura 5.

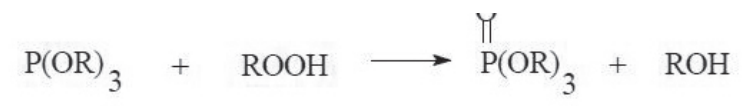

$\mathrm{ROOH}=$ hydroperoxide $\quad \mathrm{ROH}=$ alcohol

Figura 5: Mecanismo de ação dos fosfitos

\section{Procedimento Experimental}

\section{A EXTRAÇÃO DOS ADITIVOS}

Uma amostra padrão de PEAD, com aproximadamente 790 micrograma/mL de irgafos 168 e 420 micrograma/ $\mathrm{mL}$ de irganox 1076, foi moída em moinho de facas. Foi pesada a massa de $0,5000 \mathrm{~g}(+/-0,0002 \mathrm{~g})$ e em seguida esta amostra foi colocada no digestor.

Esse digestor é um sistema de síntese por microondas focalizadas e foi projetado para aumentar a habilidade de realizar reações químicas sob condições controladas e em escala de laboratório. Ele acomoda frascos de uma faixa de volume de 5 a $125 \mathrm{~mL}$ para trabalhos em condições atmosféricas, e de 10 a $35 \mathrm{~mL}$ para reações realizadas à altas pressões. Já que a massa a ser pesada é muito pequena, é importante que a amostra seja adicionada diretamente no frasco do aparelho, para que seja evitada qualquer chance de perda de material.
Esse sistema permite que sejam liberados conteúdos gasosos não desejados do digestor, para prevenir uma pressurização e falha do frasco, devido a esse conteúdo gasoso. O seu dispositivo Act-Vent libera o gás do frasco, de modo seguro, através do tubo de ventilação conectado à traseira do sistema.

A energia de micro-ondas é aplicada ao conteúdo do frasco para acelerar o processo de extração. As propriedades de absorção de micro-ondas de alguns materiais sólidos e líquidos, devido às suas características polares e iônicas, têm a capacidade de melhorar significativamente as extrações, relativamente às técnicas de aplicação de aquecimento convencionais.

Este sistema possui um sistema de produção de micro-ondas contínuo, e com uma potência selecionada pelo operador, ficando na faixa entre $0-300$ Watts.

Possui também um sistema de controle de temperatura por infravermelho, utilizando um sensor de infravermelho não invasivo, para a medida interna da temperatura do frasco. Ele fica localizado fora da cavidade e mede a temperatura do líquido dentro do frasco. O sensor é usado em um loop de informação com o sistema de controle do aparelho, para controlar a taxa de aumento de temperatura e o ponto de controle do conteúdo do frasco. A temperatura é programável entre 0 a $300^{\circ} \mathrm{C}$. Nesse aparelho, também existe a opção de agitação, através de um sistema composto por uma placa magnética, localizada abaixo da cavidade de microondas. A agitação acontece quando o campo magnético se acopla com a barra de agitação dentro do frasco.

Interessante observar que, apesar do uso de microondas, caso na amostra exista algum resíduo metálico, isso não impactará na performance do aparelho, já que este resíduo estará mergulhado em uma camada de solvente, isolando-o do meio. A estrutura da molécula do solvente utilizado impactará fortemente na geração de micro-ondas. Assim os solventes parafínicos nunca deverão ser utilizados separadamente, porque, nesses casos, a eficiência de extração será grandemente prejudicada.

Na Tabela 3, é apresentado um grupo de solventes com as suas respectivas capacidades de geração de calor, quando expostos à micro-ondas. Com base nesses dados, não foi utilizado o ciclo-hexano puro, apesar de ser considerado na literatura como um bom solvente 
para extração de aditivos em PEAD. Nesse caso, foi necessário utilizar outro solvente que pudesse gerar alta energia, como é o caso dos solventes que possuem grupamentos hidroxila em sua estrutura. Esse fato pode ser comprovado durante o desenvolvimento deste trabalho.

Foi observado que a utilização de apenas ciclohexano, ou a mistura deste com IPA, assim como quaisquer outras misturas binárias, produziram uma recuperação extremamente baixa dos aditivos no PEAD. Foram feitas inúmeras tentativas, utilizando misturas de ciclo-hexano, IPA, tolueno, diclorometano, metanol, acetato de etila e clorofórmio em composições binárias as mais variadas, sem qualquer sucesso. Alguns dados de composição ternária estão mostrados na Tabela 4. Devido aos dados obtidos, adotou-se a utilização de composições ternárias.

Tabela 3. Relação entre estrutura química e geração de calor por absorção de micro-ondas.

\begin{tabular}{|c|c|}
\hline $\begin{array}{c}\text { ABSORBÂNCIA DE } \\
\text { MICRO-ONDAS }\end{array}$ & SOLVENTE \\
\hline Alta (100W) & $\begin{array}{c}\text { DMSO,EtOH,MeOH,Pro } \\
\text { panóis,nitrobenzeno,ácido } \\
\text { fórmico, etileno glicol. }\end{array}$ \\
\hline Aédia(200W) & $\begin{array}{c}\text { Água, DMF, NMP, } \\
\text { acetonitrilametil-etil-cetona, } \\
\text { acetona, 1, 2-dicloroetano, } \\
\text { ácido acético. }\end{array}$ \\
& $\begin{array}{c}\text { Clorofórmio,diclorometano, } \\
\text { acetato de etila, xilenos, } \\
\text { benzeno, tolueno, hexano, } \\
\text { pentano,piridina,1-4dioxano. }\end{array}$ \\
\hline Baixa(300W) &
\end{tabular}

Nielsen et al. ${ }^{3}$, Bart et al. $^{4}$ e Pinto ${ }^{10}$ descrevem problemas encontrados na extração de aditivos em PEAD, levando à utilização de técnicas muito mais agressivas para esta finalidade ${ }^{10,11}$.

Usando-se misturas ternárias foi obtida substancial melhora na recuperação dos aditivos em PEAD. Na Tabela 5 e no Gráfico 1, são mostrados alguns dados relativos à extração do Irganox 1076. Nesse gráfico, podem-se observar quais destas combinações tiveram melhores resultados. $\mathrm{Na}$ Tabela 6 e no Gráfico 2, são apresentados dados relativos à extração do Irgafos 168 fosfato mais fosfito. Constatou- se que a mistura ciclo-hexano, diclorometano e isopropanol, na proporção 60:30:10, foi a que demonstrou ter o melhor poder de extração.

Tabela 4. Processo de seleção de solventes e variações na proporção da mistura.

\begin{tabular}{|c|c|c|c|c|}
\hline Solventes & $\mathrm{T}(\mathrm{oC})$ & $\begin{array}{c}\text { Tempo } \\
(\mathrm{min})\end{array}$ & $\begin{array}{c}\text { Irg1076 } \\
\mu \mathrm{g} / \mathrm{mL}\end{array}$ & $\begin{array}{l}\text { Irg168 } \\
\text { ppmm }\end{array}$ \\
\hline $\begin{array}{c}\mathrm{ciC6} / \\
\text { DCM/IPA } \\
6: 3: 1\end{array}$ & 78 & 30 & 323 & 786 \\
\hline $\begin{array}{c}\mathrm{ciC6} / \\
\text { DCM/IPA } \\
6: 3: 1\end{array}$ & 80 & 30 & 375 & 878 \\
\hline $\begin{array}{c}\text { ciC6/ } \\
\text { DCM/IPA } \\
6: 3: 1\end{array}$ & 70 & 30 & 383 & 827 \\
\hline $\begin{array}{c}\text { ciC6/ } \\
\text { DCM/ IPA } \\
6: 3: 1\end{array}$ & 75 & 30 & 363 & 865 \\
\hline $\begin{array}{c}\mathrm{ciC} 6 / \\
\text { DCM/IPA } \\
6: 3: 1\end{array}$ & 75 & 20 & 333 & 766 \\
\hline $\begin{array}{c}\mathrm{ciC} 6 / \\
\text { DCM/IPA } \\
6: 3: 1 \\
\end{array}$ & 75 & 15 & 348 & 788 \\
\hline $\begin{array}{c}\text { ciC6/ } \\
\text { DCM/IPA } \\
6: 3: 1\end{array}$ & 75 & 30 & 336 & 763 \\
\hline $\begin{array}{c}\text { ciC6/ } \\
\text { DCM/ } \\
\text { TOL } 3: 3: 4\end{array}$ & 80 & 40 & 330 & 829 \\
\hline $\begin{array}{c}\mathrm{ciC6} / \\
\text { DCM/ } \\
\text { TOL } 3: 1: 6 \\
\end{array}$ & 80 & 40 & 353 & 846 \\
\hline $\begin{array}{c}\mathrm{ciC6} / \\
\mathrm{DCM} / \mathrm{IPA} \\
6: 3: 1\end{array}$ & 80 & 40 & 368 & 865 \\
\hline $\begin{array}{c}\mathrm{ciC} 6 / \\
\text { DCM/IPA } \\
4: 4: 2\end{array}$ & 80 & 40 & 342 & 802 \\
\hline $\begin{array}{c}\mathrm{ciC} 6 / \\
\text { DCM/IPA } \\
7: 2: 1 \\
\end{array}$ & 80 & 40 & 327 & 770 \\
\hline $\begin{array}{c}\text { ciC6/ } \\
\text { DCM/ } \\
\text { TOL 6:3:1 }\end{array}$ & 80 & 40 & 341 & 814 \\
\hline $\begin{array}{c}\text { ciC6/ } \\
\text { DCM/ } \\
\text { IPA } 6: 2: 2\end{array}$ & 80 & 40 & 782 & 841 \\
\hline
\end{tabular}


Tabela 5. Rendimento do processo de extração em função do teor recuperado de Irganox 1076 em vários experimentos.

\begin{tabular}{|c|c|c|}
\hline SOLVENTES & EXP. & $\mu \mathrm{g} / \mathrm{mL}$ \\
\hline $\begin{array}{c}\mathrm{ciC} 6 / \mathrm{DCM} / \mathrm{IPA} \\
6: 3: 1\end{array}$ & 1 & 323 \\
\hline $\begin{array}{c}\text { ciC6/DCM/IPA } \\
6: 3: 1\end{array}$ & 2 & 375 \\
\hline $\begin{array}{c}\mathrm{ciC} 6 / \mathrm{DCM} / \mathrm{IPA} \\
6: 3: 1\end{array}$ & 3 & 383 \\
\hline $\begin{array}{c}\mathrm{ciC} 6 / \mathrm{DCM} / \mathrm{IPA} \\
6: 3: 1\end{array}$ & 4 & 363 \\
\hline $\begin{array}{c}\mathrm{ciC} 6 / \mathrm{DCM} / \mathrm{IPA} \\
6: 3: 1\end{array}$ & 5 & 333 \\
\hline $\begin{array}{c}\text { ciC6/DCM/IPA } \\
6: 3: 1\end{array}$ & 6 & 348 \\
\hline $\begin{array}{c}\text { ciC6/DCM/IPA } \\
6: 3: 1\end{array}$ & 7 & 336 \\
\hline $\begin{array}{c}\text { ciC6/DCM/tol } \\
3: 3: 4\end{array}$ & 8 & 330 \\
\hline $\begin{array}{c}\mathrm{ciC} 6 / \mathrm{DCM} / \mathrm{tol} \\
3: 1: 6\end{array}$ & 9 & 353 \\
\hline $\begin{array}{c}\mathrm{ciC} 6 / \mathrm{DCM} / \mathrm{IPA} \\
6: 3: 1\end{array}$ & 10 & 368 \\
\hline $\begin{array}{c}\text { ciC6/TOL/IPA } \\
4: 4: 2\end{array}$ & 11 & 342 \\
\hline $\begin{array}{c}\text { ciC6/TOL/IPA } \\
7: 2: 1\end{array}$ & 12 & 327 \\
\hline $\begin{array}{c}\text { ciC6/DCM/tol } \\
6: 3: 1\end{array}$ & 13 & 341 \\
\hline $\begin{array}{c}\mathrm{ciC} 6 / \mathrm{DCM} / \mathrm{IPA} \\
6: 2: 2\end{array}$ & 14 & 383 \\
\hline $\begin{array}{c}\text { ciC6/DCM/IPA } \\
6: 1: 3\end{array}$ & 15 & 307 \\
\hline $\begin{array}{c}\mathrm{ciC} 6 / \mathrm{DCM} / \mathrm{IPA} \\
5: 4,8: 2\end{array}$ & 16 & 313 \\
\hline $\begin{array}{c}\mathrm{ciC} 6 / \mathrm{DCM} / \mathrm{IPA} \\
6: 3,8: 3\end{array}$ & 17 & 302 \\
\hline $\begin{array}{c}\text { ciC6/DCM } \\
1: 1\end{array}$ & 18 & 307 \\
\hline $\begin{array}{c}\text { TOL/IPA } \\
9: 1\end{array}$ & 19 & 340 \\
\hline ciC6/TOL & 20 & 299 \\
\hline
\end{tabular}

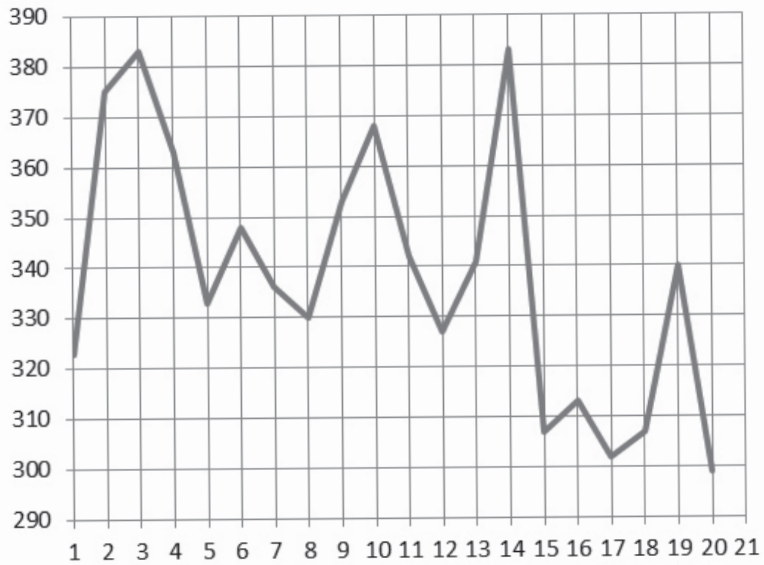

Grafico 1. Rendimento do processo de extração em função do teor recuperado de Irganox 1076.

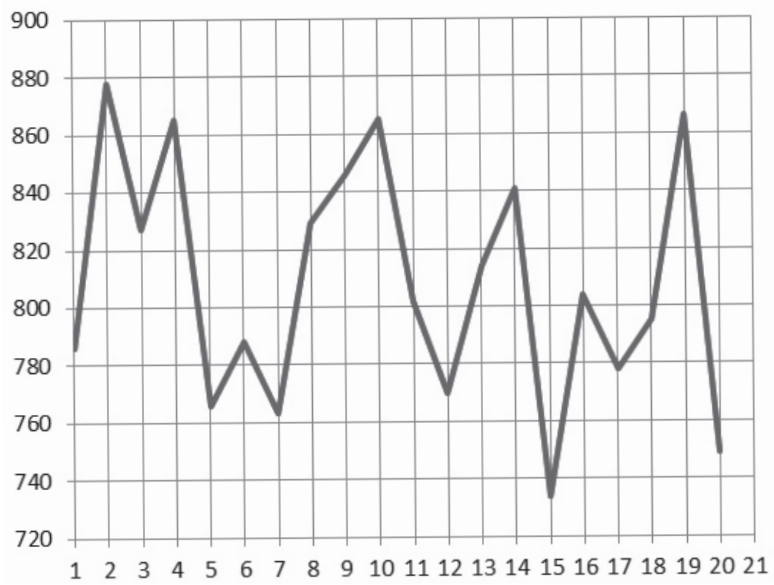

Grafico 2. Rendimento do processo de extração em função do teor recuperado de Irganox 1076.

Interessante observar que a mesma mistura de solventes é adequada tanto para a extração do irganox 1076 quanto para o irgafos 168 fosfito e fosfato.

Na Tabela7, estão mostrados os dados de recuperação dos aditivos através de refluxo com chapa aquecida, com as temperaturas de $175^{\circ} \mathrm{C}$ e $195^{\circ} \mathrm{C}$, utilizando ciclohexano, solvente apontado na literatura como o de melhor desempenho de extração e na Tabela 8 , os dados para a extração executado no digestor, para fins comparativos. 
Tabela 6. Rendimento do processo de extração em função do teor recuperado de irgafos 168 .

\begin{tabular}{|c|c|c|}
\hline SOLVENTES & EXP. & $\mu \mathrm{g} / \mathrm{mL}$ \\
\hline $\begin{array}{c}\text { ciC6/DCM/IPA } \\
6: 3: 1\end{array}$ & 1 & 786 \\
\hline $\begin{array}{c}\text { ciC6/DCM/IPA } \\
6: 3: 1\end{array}$ & 2 & 878 \\
\hline $\begin{array}{c}\text { ciC6/DCM/IPA } \\
6: 3: 1\end{array}$ & 3 & 827 \\
\hline $\begin{array}{c}\text { ciC6/DCM/IPA } \\
6: 3: 1\end{array}$ & 4 & 865 \\
\hline $\begin{array}{c}\text { ciC6/DCM/IPA } \\
6: 3: 1\end{array}$ & 5 & 766 \\
\hline $\begin{array}{c}\text { ciC6/DCM/IPA } \\
6: 3: 1\end{array}$ & 6 & 788 \\
\hline $\begin{array}{c}\text { ciC6/DCM/IPA } \\
6: 3: 1\end{array}$ & 7 & 763 \\
\hline $\begin{array}{c}\mathrm{ciC} 6 / \mathrm{DCM} / \mathrm{tol} \\
3: 3: 4\end{array}$ & 8 & 829 \\
\hline $\begin{array}{c}\mathrm{ciC} 6 / \mathrm{DCM} / \mathrm{tol} \\
3: 1: 6\end{array}$ & 9 & 846 \\
\hline $\begin{array}{c}\text { ciC6/DCM/IPA } \\
6: 3: 1\end{array}$ & 10 & 865 \\
\hline $\begin{array}{c}\text { ciC6/TOL/IPA } \\
4: 4: 2\end{array}$ & 11 & 802 \\
\hline $\begin{array}{c}\text { ciC6/TOL/IPA } \\
7: 2: 1\end{array}$ & 12 & 770 \\
\hline $\begin{array}{c}\mathrm{ciC} 6 / \mathrm{DCM} / \mathrm{tol} \\
6: 3: 1\end{array}$ & 13 & 814 \\
\hline $\begin{array}{c}\text { ciC6/DCM/IPA } \\
6: 2: 2\end{array}$ & 14 & 734 \\
\hline $\begin{array}{c}\text { ciC6/DCM/IPA } \\
6: 1: 3\end{array}$ & 15 & 804 \\
\hline $\begin{array}{c}\text { ciC6/DCM/IPA } \\
5: 4,8: 2\end{array}$ & 16 & 778 \\
\hline $\begin{array}{c}\text { ciC6/DCM/IPA } \\
6: 3,8: 3\end{array}$ & 17 & 795 \\
\hline ciC6/DCM 1:1 & 18 & 866 \\
\hline TOL/IPA 9:1 & 19 & 749 \\
\hline ciC6/TOL & 20 & 299 \\
\hline
\end{tabular}

Tabela 7. Dados de extração utilizando refluxo

\begin{tabular}{|c|c|c|}
\hline $\begin{array}{c}\text { AMOSTRA } \\
\text { (REFLUXO) }\end{array}$ & $\begin{array}{c}\text { IRGANOX 1076 } \\
\text { (MG/ML) }\end{array}$ & $\begin{array}{c}\text { IRG168-FOSFATO } \\
\text { (MG/ML) }\end{array}$ \\
\hline PEAD_ciC6_1h & & \\
\hline (175oC) & 253 & 390 \\
\hline PEAD_ciC6_1h & & \\
\hline (195oC) & 343 & 585 \\
\hline PEAD_ciC6_2h & & \\
\hline (195oC) & 250 & 404 \\
\hline
\end{tabular}

Tabela 8. Dados de Extração de aditivos pelo digestor para diferentes solventes. Nas duas primeiras linhas da coluna, foram feitas análises em duplicata. Na última a melhor condição de extração pelo digestor.

\begin{tabular}{|c|c|c|}
\hline $\begin{array}{c}\text { AMOSTRA } \\
\text { (DIGESTOR) }\end{array}$ & $\begin{array}{l}\operatorname{Irg1076} \\
(\mu \mathrm{g} / \mathrm{mL})\end{array}$ & $\begin{array}{c}\operatorname{Irg168} \\
(\mu \mathrm{g} / \mathrm{mL})\end{array}$ \\
\hline $\begin{array}{c}\text { PEAD } \\
\text { Discoveri-1 (DCM/ } \\
\text { CHCl3 - 1:1) }\end{array}$ & 181 & 272 \\
\hline $\begin{array}{c}\text { PEAD } \\
\text { Discoveri-2 (DCM/ } \\
\text { CHCl3 - 1:1) }\end{array}$ & 190 & 293 \\
\hline $\begin{array}{l}\text { PEAD_Discover } \\
\text { (IPA/ciC6 -1:1) }\end{array}$ & 295 & 440 \\
\hline $\begin{array}{l}\text { PEAD_Discover } \\
\text { (IPA/ciC6 -95:5) }\end{array}$ & 116 & 84 \\
\hline $\begin{array}{l}\text { PEAD_Discover } \\
\text { (IPA/ciC6 -10:90) }\end{array}$ & 280 & 510 \\
\hline $\begin{array}{l}\text { PEAD_Discover } \\
\text { (Tolueno) }\end{array}$ & 298 & 388 \\
\hline $\begin{array}{c}\text { PEAD_Discover } \\
\text { (ciC6/DCM/IPA } \\
\text { 60:30:10) }\end{array}$ & 396 & 733 \\
\hline
\end{tabular}

Fica evidente que a extração por micro-ondas, em condição otimizada, é muito mais efetiva do que a de refluxo, se compararmos os dados apresentados nas Tabelas 7 e 8. Outro fato positivo é a redução do tempo de extração utilizando-se esta técnica. 


\section{ANÁLISE POR HPLC}

Aparelho: Ccromatógrafo waters, modelo 2695, possui módulo de injetor automático de amostras e forno com válvula para instalação de até 3 colunas.

- Coluna: Waters, Nova-Pak C18, 3,9mm x 150mm x 4um

- Detector: UV modelo 2487 dual absorbance detector, Comprimento de onda: $200 \mathrm{~nm}$

- Fase Móvel: isocrática (85:15); Acetonitrila; B: Metanol ; Fluxo: 1,4 mL/min

- Temperatura da Coluna: $40^{\circ} \mathrm{C}$

- Tempo de análise: 8,0 minutos

- Volume de injeção: 2,0 uL

- Amostra Padrão: foi acondicionada em frascos da hach e em freezer. Antes da sua utilização ,sempre deve ser esperado que a temperatura do frasco retorne à temperatura ambiente. Os analitos poderão ficar insolúveis a baixas temperaturas. Nesse tipo de acondicionamento, praticamente ,não existe perda de solvente por evaporação. Com certeza, a baixas temperaturas e no escuro, a velocidade de degradação desses aditivos, é insignificante, mesmo na presença de clorofórmio. Todos os aditivos foram facilmente solubilizados nesse solvente.

Por questões de segurança, ele foi acondicionado sob coifa para a eliminação total de vapores orgânicos. Toda a manipulação dos solventes é feita nesse ambiente. Diariamente, é feita rotina de lavagem do injetor.

O líquido contido no frasco, após filtração, é totalmente límpido, evidenciando a eficiência da remoção dos monômeros eventualmente extraídos no processo de extração. Nessa condição, pode ser injetado no HPLC. Nas Figuras 6 e 7 são apresentados dois cromatogramas relativos à corrida das amostras padrão e de PEAD, respectivamente. Na Figura 6, tem-se o cromatograma relativo à amostra padrão de calibração, confeccionada a partir dos aditivos puros, segundo concentração mostrada na Tabela 9.

Neles pode-se observar uma boa separação entre os picos dentro de um tempo de análise bastante reduzido, o que redunda em uma alta produtividade analítica. Após o tempo de extração, o frasco é removido do aparelho
Tabela 8. Composição da Mistura Padrão de Calibração.

\begin{tabular}{|c|c|}
\hline ADITIVO & CONC. $(\boldsymbol{\mu g} / \mathbf{m L})$ \\
\hline Irganox 1010 & 306 \\
\hline Irganox 1076 & 263 \\
\hline Irgafos 168-fosfato & 188 \\
\hline Irgafos 168-fosfito & 290 \\
\hline
\end{tabular}

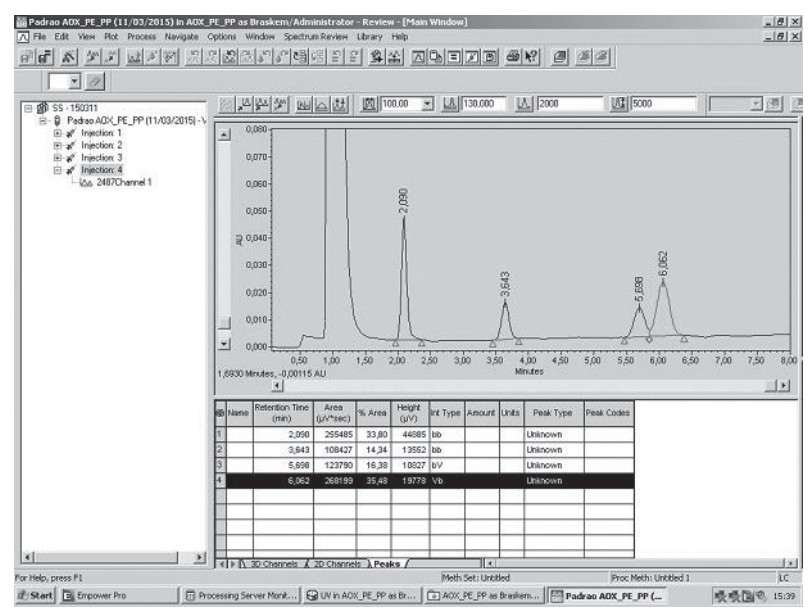

Figura 6. Cromatograma da Amostra Padrão de Aditivos.

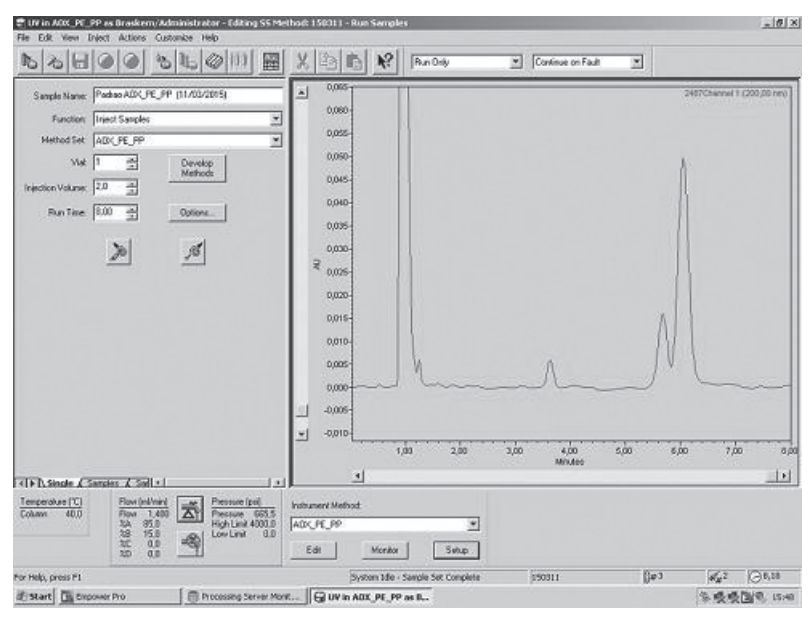

Figura 7. Cromatograma de Amostra Padrão de PEAD 
e resfriado em banho de água gelada, utilizando-se uma seringa e um cartucho para filtração.

Foi observado que a fase móvel, composta por ACN/Metanol/Água na proporção 85:14:1, melhora significativamente a separação irganox 1076 do irgafos 168-fosfito.

\section{Resultados e Discussão}

Algumas metodologias apontam que o ciclo-hexano é o melhor solvente para a extração por refluxo. Foram feitas inúmeras tentativas e não foi obtido nenhum sucesso com o seu uso, apesar de ser variada a temperatura da placa, a taxa de agitação dentro do frasco e, também, adicionando-se iso-propanol. Quando foi feito um incremento no tempo de extração para duas horas, foi observado que houve formação de um gel no extrato, sendo muito difícil a sua filtração para posterior injeção do cromatógrafo. O teores dos aditivos recuperados, conforme mostrado na Tabela 7 , não ficaram dentro da faixa esperada.

Interessante observar que, para o tempo de uma hora, a extração é muito mais efetiva, fornecendo maiores teores tanto de irganox 1076, quanto de irgafos 168. Aparentemente pode ter havido uma decomposição desses aditivos quando aquecidos por um tempo maior, ou até mesmo ter havido perda desses por aderência ao gel.

Estes fatos demonstraram que é muito mais produtivo investir em melhorar a taxa de extração, pela utilização da técnica de micro-ondas. Usando-se esta técnica, foram realizados vários testes com solventes puros, inclusive $o$ tolueno, misturas binárias desses e variando-se também a temperatura e o tempo de extração. A utilização do tolueno, solvente sabidamente agressivo na dissolução de poliolefinas, mesmo misturados a outros solventes, não gerou resultados promissores. Só foi obtido algum aumento na taxa de extração, quando passaram-se a utilizar misturas ternárias. Alguns dados desse período do trabalho estão mostrados na Tabela 8 .

Ficou caracterizado que o uso de ciclo-hexano puro não é produtivo, conforme as informações contidas na Tabela 3. Mesmo fazendo-se misturas com outros solventes que possuem pontes de hidrogênio, não foi possível obter resultados satisfatórios. Só a partir da incorporação do diclorometano na mistura de extração, obtiveram-se melhores resultados.

O objetivo deste trabalho sempre foi o de buscar um aumento na sinergia deste processo extrativo. Este estudo teve uma primeira fase: seleção dos solventes, depois passou-se a averiguar a melhor taxa de combinação entre eles e, finalmente, a melhor temperatura para a extração. Só foram obtidos bons resultados, a partir do uso de composições ternárias de solventes. Depois dessa fase, foi possível otimizar o tempo de extração, com foco no menor tempo possível, desde que não fosse prejudicada a eficiência de recuperação dos aditivos em estudo.

Cabe ressaltar que a tampa de plástico, do frasco do Discover, sofreu forte desgaste, provavelmente, devido ao uso de solventes agressivos para a sua composição. Para evitar esse fenômeno, passou-se a secá-la, logo após a extração, para diminuir o tempo de contato destes solventes com a tampa. Importante comunicar esse fato ao fabricante para que ele possa desenvolver um material mais inerte na confecção desta tampa.

\section{Conclusão}

Os dados apresentados neste trabalho mostram que é possível a utilização da técnica de extração por microondas na extração de aditivos em PEAD, não sendo a única possibilidade, mas uma alternativa bastante promissora.

\section{Referências bibliográficas}

1. Grosclaude, G., 1999. L'eau, Mileu naturel et maitrise (Tome 1), INRA Edition, Paris.

2. Lacaze, J.C., 1993,La degradation de l'environnement côtierconséquences écologiques, Ed Masson, Paris.

3. Characterization of plastic packinging additivies: Food contact, stability and toxicity, Lahimer, M.C., Ayed, N., Horriche, J., Belgaied, S., Arabian Journal of Chemistry (2014), King Saud University, july, 2013.

4. Nielsen, R., "Extraction and Quantification of Polyolefin Additivies", Journal of liquid Chromatography, Vol.14, \#3, 1991.

5. Bart, J.C.J.Polymer Additive analytics ; Industrial practice and case studies. In: Quality of food contact Polymer, first ed. Firenze, Italy, pp. 249-316.

6. Nielsen, R.C., Overview of Polylefin Additive Analysis, Waters Chromatography Division, Millipore Corporation., International GPC Symposium, oct, 1989.

7. Bart, J.C.J., “ Additivies in Polymers Industrial Analysis and 
Applications", Wiley.

8. Rapid Polyolefin Additive Separation Using a Waters Nova-Pak C18 column, Waters Applications Notes, Waters Corporation, Milford, Massachussets, USA.

9. ASTM D 7210-06, "Standard Practice for Extraction of Additivies in Polyolefin Plastics".

10. Pinto, A.M.,"Quantitative Analysis of Antioxidants from High Density Polyethylene (HDPE) by off-line Supercritical Fluid Extraction Coupled High Performance Liquid Chromatography, Virginia Polytechnic Institute State University, Blacksburg, aug, 1997.

11. Peinado, C.A.,Corrales,T.A.,Garcia,M.J.,Catalina,F.A.,"Chemilu minescence from poly(styrene-b-ethylene-co-butylene-bstyrene) (SEBS) block copolymers, Carmen Peinado a, ${ }^{*}$, Teresa Corrales a, M. Jesu' s Garc1'a-Casas a, Fernando Catalina a,Valentı'n Ruiz Santa Quiteria b, M. Dolores Parellada b, Instituto de Ciencia y Tecnologia de Polımeros, CSIC, Juan de la Cierva 3, 28006 Madrid, Spain,oct-2005.

\section{Augusto O.Cardoso}

BRASKEM, UNIB-4/DCX-PE9-LCQ, Duque de Caxias-RJ, Brasil.

E-mail: aocrj@yahoo.com.br 